\title{
Sub GV/cm Terahertz Radiations from Relativistic Laser-Solid Interaction via Coherent Transition Radiation
}

\author{
W. J. Ding ${ }^{1 *}$ (丁文君) and Z. M. Sheng ${ }^{2,3,4}$ (盛政明) \\ ${ }^{1} A * S T A R$ Institute of High Performance Computing, Singapore 138632 \\ ${ }^{2}$ SUPA, Department of Physics, University of Strathclyde, Glasgow G4 ONG, UK \\ ${ }^{3}$ Key Laboratory for Laser Plasmas (MoE) and Department of Physics and Astronomy, \\ Shanghai Jiao Tong University, Shanghai 200240, China \\ ${ }^{4}$ Collaborative Innovation Center of IFSA, Shanghai Jiao Tong University, Shanghai 200240, \\ China \\ *Email:dingwj@ihpc.a-star.edu.sg
}

\begin{abstract}
Broadband terahertz $(\mathrm{THz})$ radiation with extremely high peak power, generated by the interaction of femtosecond laser with a thin solid target, has been investigated via particle-in-cell simulations. The spatial (angular) and temporal profiles of the $\mathrm{THz}$ radiation reveal that it is caused by the coherent transition radiation emitted when laser-produced hot electrons pass through the front or rear surface of the target. Dependence of the $\mathrm{THz}$ radiation on laser and target parameters is studied; it is shown to have strong correlation with hot electron production. The THz radiation conversion efficiency can be as high as a few times of $10^{-3}$. This radiation is not only a potentially high power THz source, but may also be used as a unique diagnostic of hot electron generation and transport in relativistic laser-solid interactions.
\end{abstract}

PACS numbers: 41.60.Dk, 52.25.Os, 52.38.Dx, 52.38.-r, 52.40.Mj, 52.59.-f, 52.59.Ye, 52.65.-y, 52.65.Rr 


\section{INTRODUCTION}

Laser plasma interaction is a promising technology towards table-top extremely powerful terahertz $(\mathrm{THz})$ sources $^{1,2}$ with a peak power over a gigawatt, because it overcomes the damage limitation of other optical material based $\mathrm{THz}$ sources ${ }^{3}$. Another kind of strong THz sources is based on traditional linear accelerators. By bending a relativistic electron bunch in a magnetic field or sending it through certain structures, $\mathrm{THz}$ sources with an electric field of $70 \mathrm{MV} / \mathrm{cm}$ and an energy exceeding $100 \mu \mathrm{J}$ can be generated by a $10 \mathrm{GeV}$ of electron beam ${ }^{4}$. However, these accelerator based $\mathrm{THz}$ sources are often large and expensive; their accessibility is limited. Laser plasma based $\mathrm{THz}$ sources have attracted much attention recently, and various mechanisms have been reported on the interaction of laser with solid ${ }^{5-7}$ or gas targets ${ }^{8-13}$. The radiation mechanisms are often different for interactions with solid and gas targets. Radiation efficiency and peak $\mathrm{THz}$ radiation power with solid targets can be much higher than those of gas targets, since more laser energy can be deposited into a small plasma volume in this case and more energetic electrons are involved in the radiation process.

In a previous study $^{14}$, we have shown that strong $\mathrm{THz}$ radiation, with a peak electric field as high as hundreds of $\mathrm{MV} / \mathrm{cm}$, can be obtained with a solid target irradiated by a femtosecond laser pulse of relativistic high intensity. It is attributed to the coherent transition radiation $(\mathrm{CTR})^{15}$ by hot electrons produced during the laser-solid interaction, when they cross the front and rear surfaces of the target. In the scheme of producing $\mathrm{THz}$ radiation by electron beams produced by a conventional accelerator, e.g., by sending an electron beam through an aperture ${ }^{4,16}$, the radiation field strength reaches only tens of $\mathrm{MV} / \mathrm{cm}$. With the method of ultrashort laser-solid interaction, although most electrons have a moderate energy around $1 \mathrm{MeV}$ or less, the $\mathrm{THz}$ radiation can reach hundreds of $\mathrm{MV} / \mathrm{cm}$ and higher. As the electron bunches typically have an ultrashort duration close the laser duration, transition radiation from the electron bunches becomes coherent ${ }^{15}$, with a power proportional to $N^{215}$, where $N$ is the number of hot electrons. In laser-solid interaction, the electron charge in an electron bunch can be as high as $\mathrm{nC}$, which is much higher than the charge of an accelerated electron beam in a traditional accelerator ${ }^{16}$.

In this work, we present detailed studies on the features of the $\mathrm{THz}$ radiation in high power laser-solid interaction via CTR. The spatial (angular) and temporal profiles of the radiation and their relation with laser and plasma parameters will be examined. A theoretical model is given in Sec. II. In real situation of laser-solid interactions, the $\mathrm{THz}$ radiation power, frequency spectra, angular distribution depend upon the laser and plasma parameters, which can only be determined by selfconsistent numerical simulations such as particle-in-cell (PIC) simulations. Detailed numerical results from PIC simulations will be presented in Sec. III, where a comparison with some recent experimental observations is made. A summary is given in Sec. IV.

\section{THEORY OF COHERENT TRANSITION RADIATION BY HOT ELECTRONS}

Transition radiation of electromagnetic waves is found when a charged particle crosses the interface of two different media or travels in a non-uniform media ${ }^{17}$. In the incidence plane, the energy spectrum of the transition radiation by a single electron passing through a target surface can be written as ${ }^{15}$ :

$\frac{d^{2} \mathcal{E}}{d \omega d \Omega}=\frac{e^{2}}{\pi^{2} c}|S(\beta, \varphi, \phi)|^{2}$,

where $e$ is the electron charge, $c$ is the light velocity in free space, $\beta$ is the electron velocity normalized to $c, \varphi$ is the electron injection direction, $\phi$ is the observation direction, and

$S(\beta, \varphi, \phi)=\frac{\beta \cos \varphi(\sin \phi-\beta \sin \varphi)}{(1-\beta \sin \phi \sin \varphi)^{2}-(\beta \cos \phi \cos \varphi)^{2}}$.

We plot the angular distributions of intensity spectrum of the transition radiation in Fig.1. In subplot (a), the electron injection direction is $20^{\circ}$; it can be seen 
that the radiation vanishes near this angle. Two asymmetric peaks are found at the two sides away from this angle, where the larger peak locates at the electron injection side. When the electron has low energy, e.g., $0.2 \mathrm{MeV}$, the radiation is emitted in a large angular range, and is strongest along the target surface as shown in Fig. 1(a). When the electron carries larger kinetic energy, the radiation becomes more collimated and close to the electron injection direction. Figure 1(b) shows the transition radiation distributions when a mildly relativistic electron, with $E_{\mathrm{k}}=0.5 \mathrm{MeV}$, is injected with different angles. When the angle increases, the strongest emission direction shifts from near the electron injection direction to the along-target direction, such as $\varphi=40^{\circ}$. With further increase of the incidence angle $\varphi$, the radiation becomes weaker and disappears at $\varphi=90^{\circ}$.

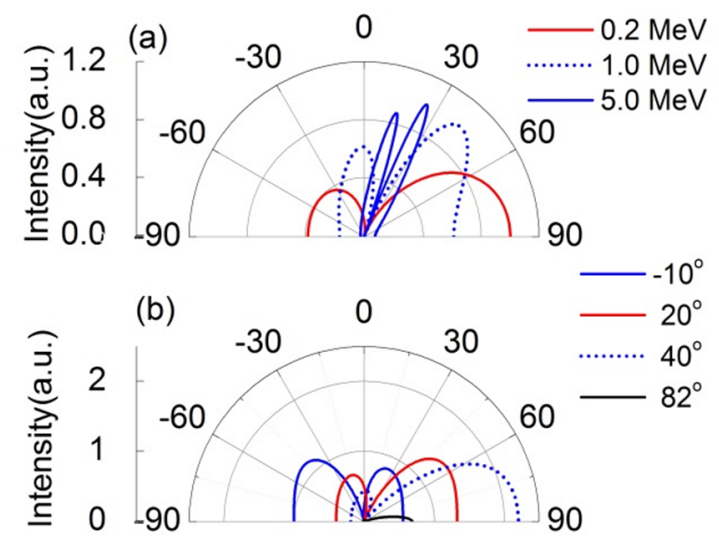

FIG. 1. (Color online) Angular $(\phi)$ intensity distributions of transition radiation by a single electron crossing a dense plasma-vacuum interface. (a) For the case of the incident angle of a test electron with $\varphi=20^{\circ}$ and different energies $E_{\mathrm{k}}$, where the distributions are normalized to their own maximums, which are 0.735 , 2.794, and 31.08 for $E_{\mathrm{k}}=0.2,1.0$, and $5.0 \mathrm{MeV}$, respectively. (b) For the case of an electron energy with $E_{\mathrm{k}}=0.5 \mathrm{MeV}$ under different incident angles.

In laser plasma interaction, huge amount of hot electrons are involved. Therefore, radiation coherence between the electrons needs to be considered. The energy spectrum of CTR by an electron beam passing through a target surface is given by ${ }^{15}$ : $\frac{d^{2} \mathcal{E}_{C T R}}{d \omega d \Omega}$

$=\frac{e^{2} N(N-1)}{\pi^{2} c}\left|\int S(\beta, \varphi, \phi) f(\tau, \boldsymbol{\rho}, \boldsymbol{v}) e^{i \omega \tau-i \boldsymbol{q} \cdot \boldsymbol{\rho}} d \tau d \boldsymbol{\rho} d \boldsymbol{v}\right|^{2}$,

where $f(\tau, \boldsymbol{\rho}, \boldsymbol{v})$ is the distribution function of electrons in an electron bunch, which is a function of time, space, and velocity. Even though Eq. (3) cannot be solved easily, it is evident that the integration in Eq. (3) will become finite when the electron bunch duration $\tau_{b}<1 / \omega_{T H z}$. In this case, the radiation is coherent, and its intensity is proportional to $N(N-1)$.

In the interaction of relativistic laser pulses with solid targets, the charge of hot electrons in a bunch can reach $\mathrm{nC}$, which is usually much higher than the case of a conventional accelerator, although the laser produced electrons have broad energy spread and relatively low average energy. Increasing the number of hot electrons would be an efficient way to obtain strong CTR. Since the radiation is coherent, one can demonstrate the radiation from self-consistent PIC simulations. In the following section, we present CTR from PIC simulation of laser-solid interaction. The results are then compared with some previous related experimental observations.

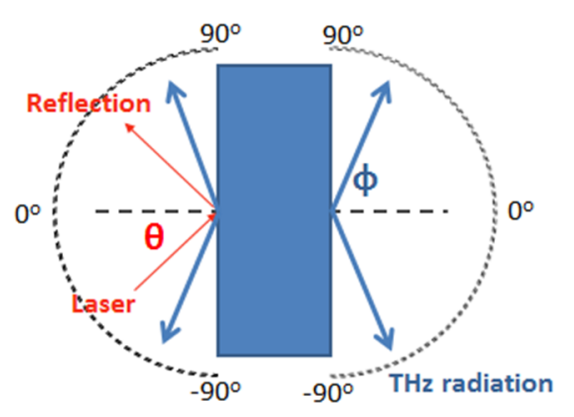

FIG. 2 Schematic of laser-solid interaction, hot electron generation, and $\mathrm{THz}$ radiation.

\section{SIMULATION RESULTS}

As mentioned in the previous section, self-consistent PIC simulation provides an accurate description of CTR as a function of the laser and plasma parameters, which can help to guide future experimental studies. This is due 
to the fact that the key parameters determining CTR such as number of energetic electrons, bunch duration, their angular distribution and energy spectrum all depend upon the laser and plasma parameters.
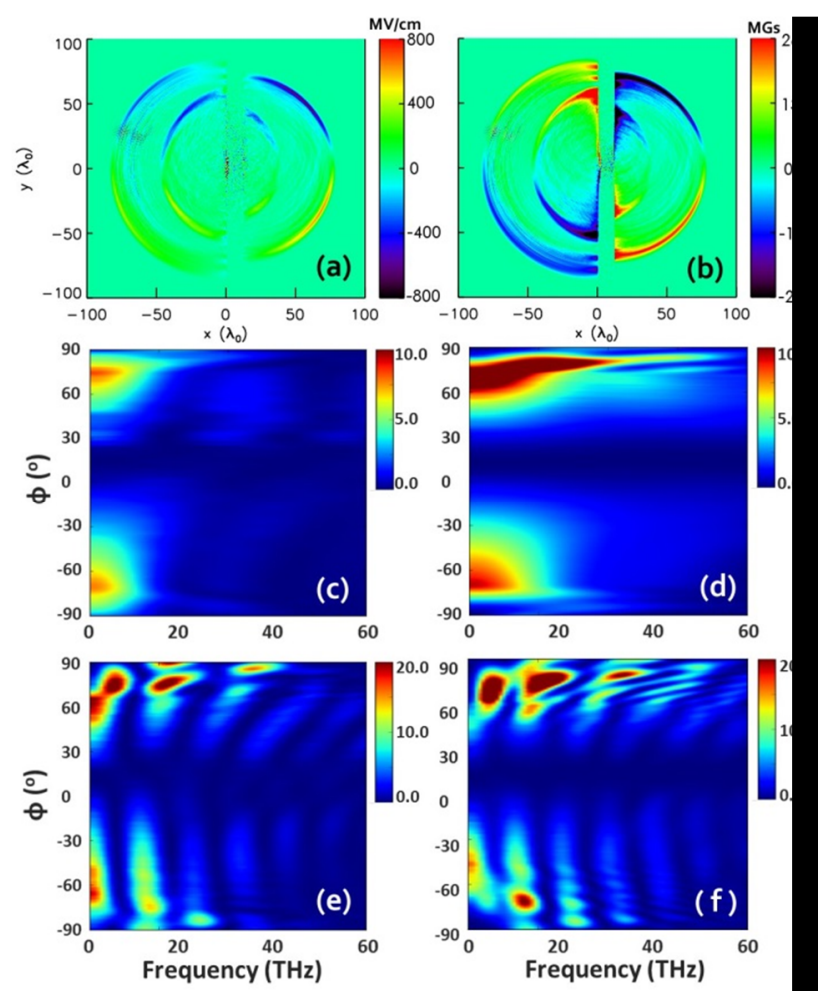

FIG. 3. (Color online) (a) and (b) are snapshots of electric field $E_{y}$ and magnetic field $B_{z}$ (averaged in a laser cycle), respectively. (c) and (d) are spectra of the first pulse of backward and forward radiations (FFT of $B_{z}$ ) at the left and right hand side of the target, respectively. (e) and (f) show spectra of the entire time series of backward and forward radiations. The laser pulse is with $a_{0}=0.6$ (intensity $I_{0}=7.7 \times 10^{17} \mathrm{~W} / \mathrm{cm}^{2}$ ), incidence angle $\theta=30^{\circ}$, width of laser spot $w_{0}=4 \mu \mathrm{m}$, and duration $T=30 \tau_{0}=80 \mathrm{fs}$; The plasma has a density scalelength of $L=0.4 \lambda_{0}$.

In the simulation, we have taken a laser pulse described by the normalized vector potential of laser field given by $a=e A / m_{e} c^{2}=a_{0} \sin ^{2}\left(\frac{\pi t}{T}\right) \exp \left(-\frac{y^{2}}{w_{0}^{2}}\right)$, where $a_{0}$ is related to the laser intensity $I \lambda_{0}^{2}=a_{0}^{2} \times 1.37 \times$ $10^{18} \mathrm{~W} / \mathrm{cm}^{2} \mu \mathrm{m}^{2}$. The full laser duration $T=30 \tau_{0}$, which is about $80 \mathrm{fs}$ for a laser with $\lambda_{0}=800 \mathrm{~nm}$ or $40 \mathrm{fs}$ in fullwidth-half-maximum (FWHM), where $\tau_{0}$ is a laser cycle. The laser spot is Gaussian profiled with FWHM radius
$w_{F W H M}=2 \sqrt{\ln 2} w_{0}=8.3 \lambda_{0}$. The laser is obliquely incident in 30 degrees onto the target with p-polarization, so that hot electrons can be produced efficiently. In the simulations plasma density exponentially increases as $n(x)=n_{0} \exp (-x / L)$ from $n_{0}$ to $10 n_{\mathrm{c}}$, where $L$ is the scalelength, $n_{0}=0.1 n_{\mathrm{c}}$ and $n_{\mathrm{c}}=m \omega_{0}^{2} / 4 \pi e^{2}$ is the critical density, which is $1.74 \times 10^{21} \mathrm{~cm}^{-3}$ for a laser with $\lambda_{0}=800 \mathrm{~nm}$. If not specified, the plasma slab is $D=10 \lambda_{0}$ thick in the high density area with $10 n_{\mathrm{c}}$ in density and $200 \lambda_{0}$ wide. Figure 2 shows the schematic of the simulations and measurements. In the following, we will first show the characteristics of the $\mathrm{THz}$ radiations generally, including their angular distributions and frequency spectra. Then the effects of laser parameters (e.g., intensity and laser spot size) and plasma parameters (e.g., target thickness and preplasma conditions) on $\mathrm{THz}$ radiation will be discussed in details.

\section{A. Angular distributions and spectra of the $\mathbf{T H z}$} radiations

Snapshots of the typical spatial distribution of radiation due to the laser produced hot electrons are plotted in Fig. 3(a) and 3(b). Forward (transmitted) and backward (reflected) radiations are emitted from the target front and rear surfaces, respectively. There are sequential radiation peaks, where the electric field can be as high as hundreds of $\mathrm{MV} / \mathrm{cm}$ at a distance of $50 \mu \mathrm{m}$ from the target. The time delay between the first and second peaks is slightly longer than $2 D / c$, which is comparable to the time for hot electrons to travel forth and backwards through the target. Under an incident angle of 30 degrees, the radiation amplitude at the upper side is also higher than that at the lower side, in addition to the fact that the fields at the two sides have opposite signs. Around an angle of 15 degree, the radiation fields vanish.

Figures 3(c) -(f) show the spectra of backward and forward electromagnetic fields, which are detected $100 \mu \mathrm{m}\left(125 \lambda_{0}\right)$ away from the laser irradiated spots in the front and rear surfaces of the target. Note that the distance is long enough so that the detected fields are really radiated fields, which are not affected by quasi-static 
fields produced around the target surface. Because multiple pulses are emitted, we perform FFT on the first pulses and the entire time series of the radiation, separately. Spectra of the first pulses for backward and forward radiations are shown in Fig. 3(c) and 3(d) respectively, while the spectra of the entire time series are shown in Fig. 3(e) and 3(f). Broadband radiations with frequency around and less than $40 \mathrm{THz}$ are detected. Both plots of backward and forward radiations show that the radiations are emitted in a large angular range, and the upper (with positive angles) and lower (with positive angles) sides are asymmetric with higher radiation power found at the upper side.

This asymmetric distribution originates from the oblique ejection of hot electrons under the oblique incidence of laser light. Note that $0^{\circ}$ and $30^{\circ}$ are the target normal and specular reflection direction, respectively; hot electrons produced from the laser-solid interaction are emitted mainly between these two directions according to a previous theory ${ }^{18}$. Most hot electrons are ejected around $\sim 17^{\circ}$, which leads to a hollow zone of $\mathrm{THz}$ radiation around this angle. This is reiterated in Fig. 4 (see below).

The broad angular distributions of the radiation can be attributed to the relatively low electron energy of the hot electrons. With these laser setup parameters, the average kinetic energy of the hot electrons is generally at the order of a hundred $\mathrm{keV}$. The transition radiation of a single electron with moderate energy, such as $E_{\mathrm{k}}=0.5 \mathrm{MeV}$, is emitted in a wide angle range according to Fig. 1(b). Furthermore, as hot electrons in laser plasma interactions have a divergence angle and large energy spread, typically with a quasi-thermal energy distribution, this naturally leads to broad angular distributions of the produced CTR. Note that the peak power of the radiation at the rear side of the target is higher than that at the front side, suggesting that there are more electrons with higher energy passing through the rear surface, which will be shown in Sec. III C. Stronger than the backward radiation, the forward radiation, shown in Fig. 3(d), has a minimum zone around $15^{\circ}$ as well, which is about the direction of transmitted hot electrons.
Modulations of the spectra in frequency, seen in Figs. 3(e) and 3(f), are due to the multiple pulses of the radiations, while the separation between the neighboring peaks of the spectrum modulations depends on the time interval of the pulses, notei.e., depends on the target thickness. For example, in Fig. 3(e), it is $f_{0} \leqslant c / 2 D=17 \mathrm{THz}$. Modulations of the spectra in angle, particularly at large angles, are observed in Figs. 3(e) and 3(f) as well, which is due to the superposition of the forward and backward emissions when they meet at the two ends of the target.

\section{B. Effects of the incident laser intensity and spot size}

According to the theory of transition radiation, fast electrons with a stronger energy will emit more collimated transition radiation. Therefore, we explore this with higher laser intensities. Angular distributions of energy for both the $\mathrm{THz}$ radiation and the reflected laser in front of the thin target are plotted in Fig. 4. Since the reflected laser fields and $\mathrm{THz}$ fields superimpose with each other at the front side of the target, they can be separated each other in the following way. The energy for THz radiation is calculated by FFT of the fields detected at $100 \mu \mathrm{m}$ away from the laser irradiated spot center, and is then summed up from 0 to $100 \mathrm{THz}$ in frequency. Similarly, the energy for the reflected laser energy is obtained by summing up from 300 to $450 \mathrm{THz}$, which is 0.8 to 1.2 times of the laser frequency.

The emissions are not collimated because the produced hot electrons have a Boltzmann energy distribution, and most of them are at non-relativistic velocities. As shown in Fig. 4, when the laser intensity increases from a few times of $10^{17}$ to $10^{19} \mathrm{~W} / \mathrm{cm}^{2}$, the strongest radiation stays around large angles along the target surface, i.e., near $\pm 90^{\circ}$. As mentioned earlier, the minimum radiation appears around the ejection directions of most hot electrons. As the laser intensity increases, Fig. 4 shows that the angle for the minimum radiation increases from about $13^{\circ}$ to $22^{\circ}$ for both the backward and forward radiations. This agrees with the theory by Sheng et al. ${ }^{18}$, which suggests that the ejecting angles of fast electrons increase with the fast electron energy (and therefore with the incident laser intensity). 
(a)

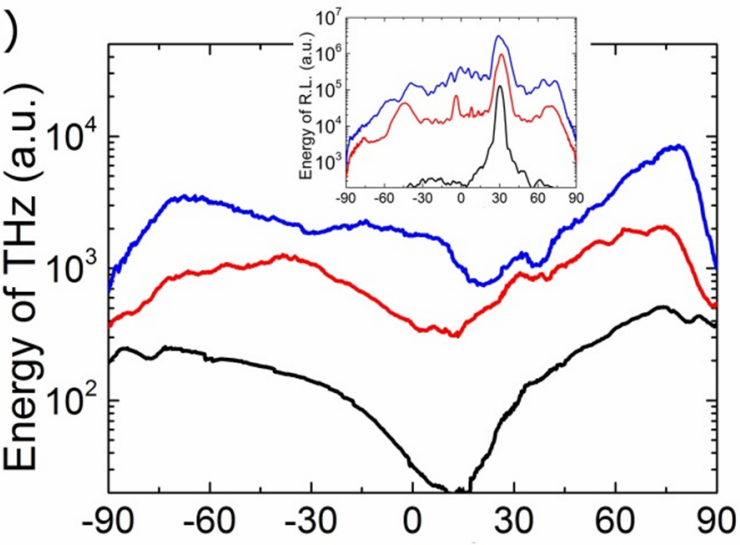

(b)

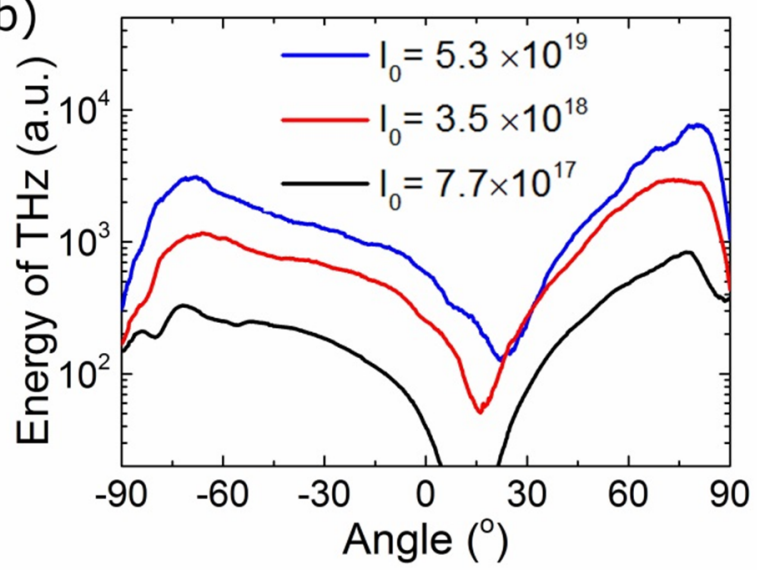

FIG. 4. (Color online) Angular distributions of energy for (a) backward and (b) forward $\mathrm{THz}$ radiations. Laser intensities are $7.7 \times 10^{17}, 3.5 \times 10^{18}$ and $5.3 \times 10^{19} \mathrm{Wcm}^{-2}$ for black, red, and blue lines, respectively. Inset in (a) shows the angular distributions of reflected laser energy. Other parameters follow Fig. 3. THz radiation energy is calculated from 0 to $100 \mathrm{THz}$. Reflected laser energy is summed up from $300 \mathrm{THz}$ to $450 \mathrm{THz}\left(0.8\right.$ to $\left.1.2 \omega_{0}\right)$.

For fixed laser intensity, when the laser is incident in different angles $\theta$, the $\mathrm{THz}$ radiation energy changes since fast electrons are emitted in different directions and the absorption rate of the laser is also different. Figure 5 shows the angular distributions of $\mathrm{THz}$ radiation energy for different $\theta$. For all cases in Fig. 5, we fix the target preplasma scalelength to be $L=0.4 \lambda_{0}$, which is optimal under an incident angle of $\theta=30^{\circ}$ (or the maximum laser absorption with lowest reflection $)^{19,20}$. As the incident angle changes from $20^{\circ}$ to $60^{\circ}$, the $\mathrm{THz}$ emission power varies. Especially for the large incident angle of $\theta=60^{\circ}$, the emission is greatly reduced for more than an order of magnitude due to the large ejecting angle of hot electrons and small laser absorption rate. In addition, for a large incident angle, some hot electrons are emitted along the target surface ${ }^{21}$, but these hot electrons do not contribute to the generation of CTR as discussed above when $\varphi=90^{\circ}$ in formula (2). The angle for the minimum $\mathrm{THz}$ emission moves from around $10^{\circ}$ to $20^{\circ}$ when the incident angle increases. In general, the angular distributions for $\mathrm{THz}$ radiation under different laser intensities are quite similar.
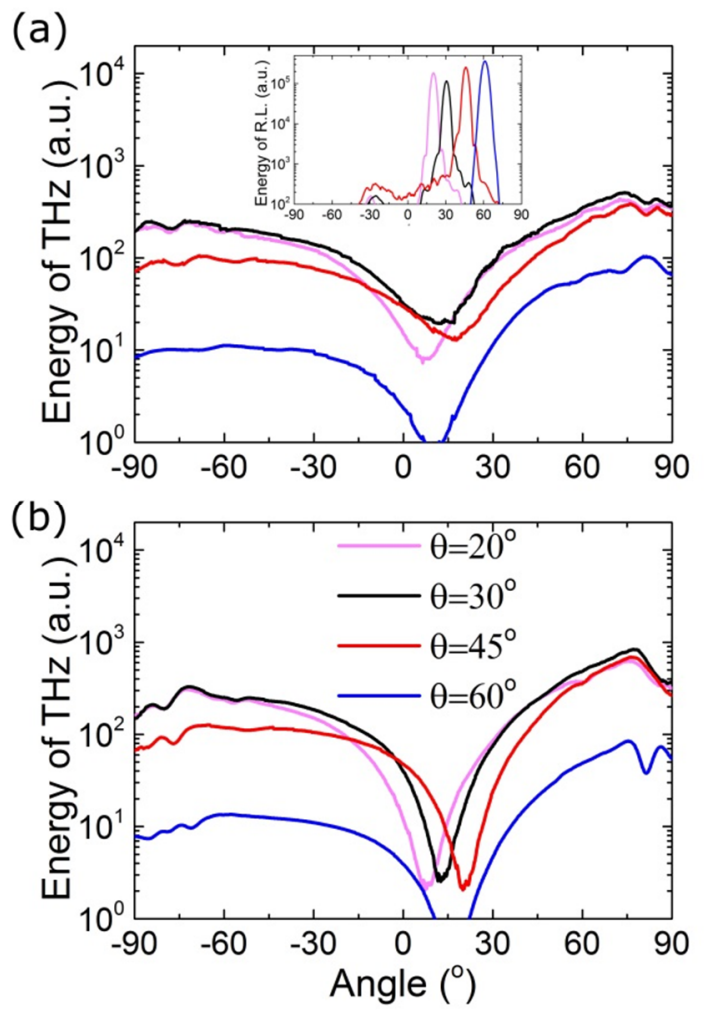

FIG. 5. (Color online) Angular distributions of energy for (a) backward and (b) forward $\mathrm{THz}$ radiations. Incidence angles are $\theta=20^{\circ}, 30^{\circ}, 45^{\circ}, 60^{\circ}$ for pink, black, red and blue lines, respectively. Inset in (a) shows the angular distributions of the reflected laser energy. Other parameters are the same as those shown in Fig. 3.

Another way to enhance the incident laser energy is by changing the laser spot size. This changes the $\mathrm{THz}$ radiation in certain direction in two ways. First, it leads to the change of the number of hot electrons. Second, it changes the $\mathrm{THz}$ radiation propagation. For the latter, 
since the focused laser spot size and corresponding electron beam diameters at the target surfaces are usually smaller than the corresponding $\mathrm{THz}$ wavelength, a large laser spot size can obviously make the $\mathrm{THz}$ radiation more collimated. THz radiations generated by lasers with different spot sizes are illustrated in Fig. 6. Comparing to the spectra in Figs. 3, it is found that the asymmetry of the $\mathrm{THz}$ radiations between the upper and lower sides becomes larger, when the waist of the laser is increased to $w_{0}=16 \mu \mathrm{m}$. The radiation at the upper side is much higher than that at the lower side, as shown in Figs. 6(a) and 6(b). The enhanced radiation is found at small angles, i.e., close to the ejecting angle of hot electrons. For example, at emission angles $-60^{\circ}, 40^{\circ}$, and $80^{\circ}$, the energy of the backward radiations with $w_{0}=16 \mu \mathrm{m}$ is $0.58,5.8$, and 1.3 times of the case with $w_{0}=4 \mu \mathrm{m}$, respectively. Overall, with a larger laser spot, the emissions become more collimated along certain directions. In general, when the laser energy is fixed, oversized laser spot may lead to reduced laser absorption and low hot electron energy, and subsequently, low $\mathrm{THz}$ radiation.
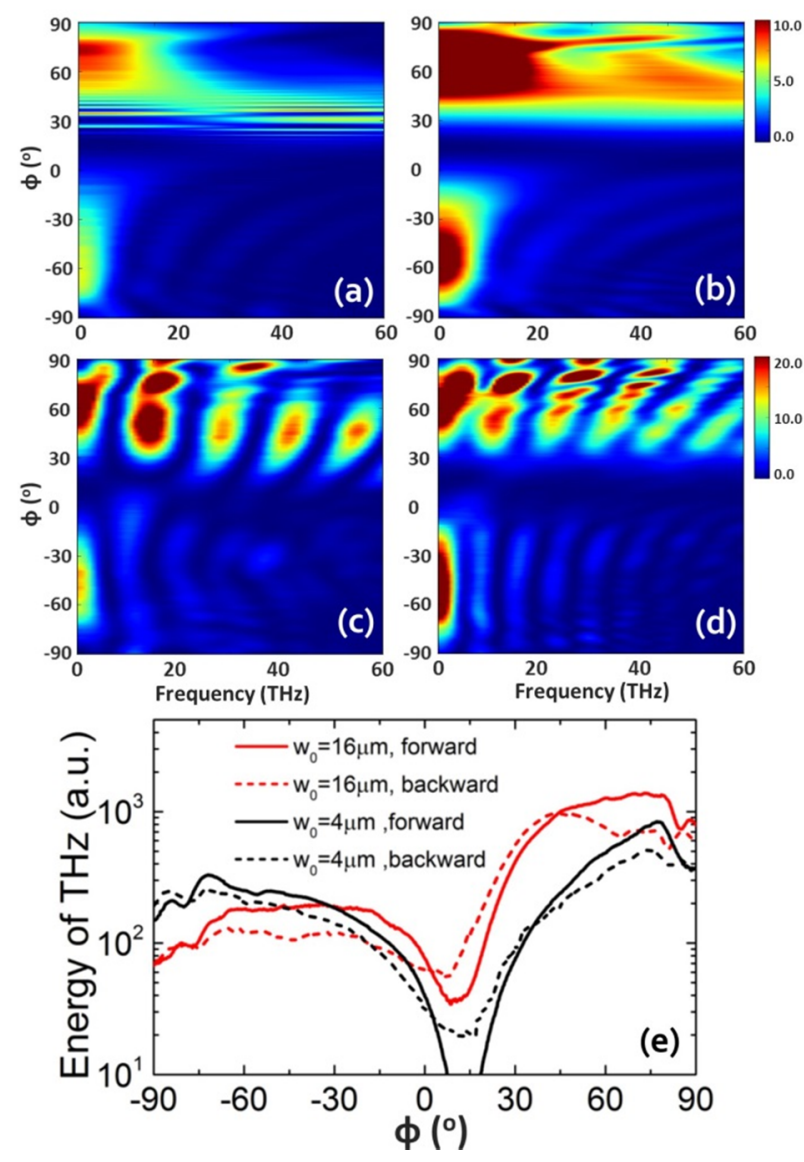

FIG. 6. (Color online) (a) and (b) are spectra of the first pulse of backward and forward $\mathrm{THz}$ radiations, respectively, with laser spot $w_{0}=16 \mu \mathrm{m}$. (c) and (d) show spectra of the entire time series of backward and forward radiations. (e) Angular distributions of energy of the radiations. Other parameters follow Fig. 3.

\section{Preplasma effects}

In ultrashort laser interaction with solid targets, the preplasma condition, in particular, the preplasma density scalelength in the front of the target is a key parameter for hot electron production, including their number and average energy. The preplasma can also significantly change the hot electron transport after the laser interaction. Thus the preplasma scalelength can play a significant role for $\mathrm{THz}$ radiation. Hot electrons are produced most efficiently by collisionless collective effects, such as resonance absorption ${ }^{22}$, vacuum heating $^{23,24}$, two-plasmon decay instability ${ }^{22}$, and ponderomotive force acceleration ${ }^{25}$. Resonance absorption occurs when the laser is obliquely incident with p-polarization, and preferably with small preplasma of the target, such as less or around a laser wavelength. Vacuum heating occurs when the target has a sharply bounded surface that the scalelength of the preplasma shorter than the plasma skin depth $L \leq c / \omega_{\mathrm{p}}$. Two-plasmon decay usually requires relatively large preplasma. Ponderomotive force acceleration grows with the laser intensity and dominates when the laser is relativistically intense and the preplasma is properly large. As these schemes are dependent upon the scale of the preplasma, we study the effect of preplasma on the generation of $\mathrm{THz}$ radiation. Such results can help identify the most effective scale in generating strong $\mathrm{THz}$ radiation. The preplasma is practically determined by the contrast ratio of laser system $^{26}$.

Figure 7(a) shows how the $\mathrm{THz}$ radiation intensity varies with the scalelength of the preplasma. With incident angle $\theta=45^{\circ}$, an optimal scalelength exists near $L=0.4 \lambda_{0}$ for preplasma, at which the strongest $\mathrm{THz}$ radiation is generated. Under the given laser conditions, the major mechanism of producing hot electrons is 
resonance absorption with medium-sized preplamsa ${ }^{27}$. For resonance absorption, there is an optimal scalelength of preplasma for the maximum absorption ${ }^{28}$. In the case with incident angle $45^{\circ}$, the optimal scalelength is around $L=0.4 \lambda_{0}$. An optimal scalelength of preplasma occurs mainly due to the fact that the number of produced hot electrons rather than their average energy is the largest.

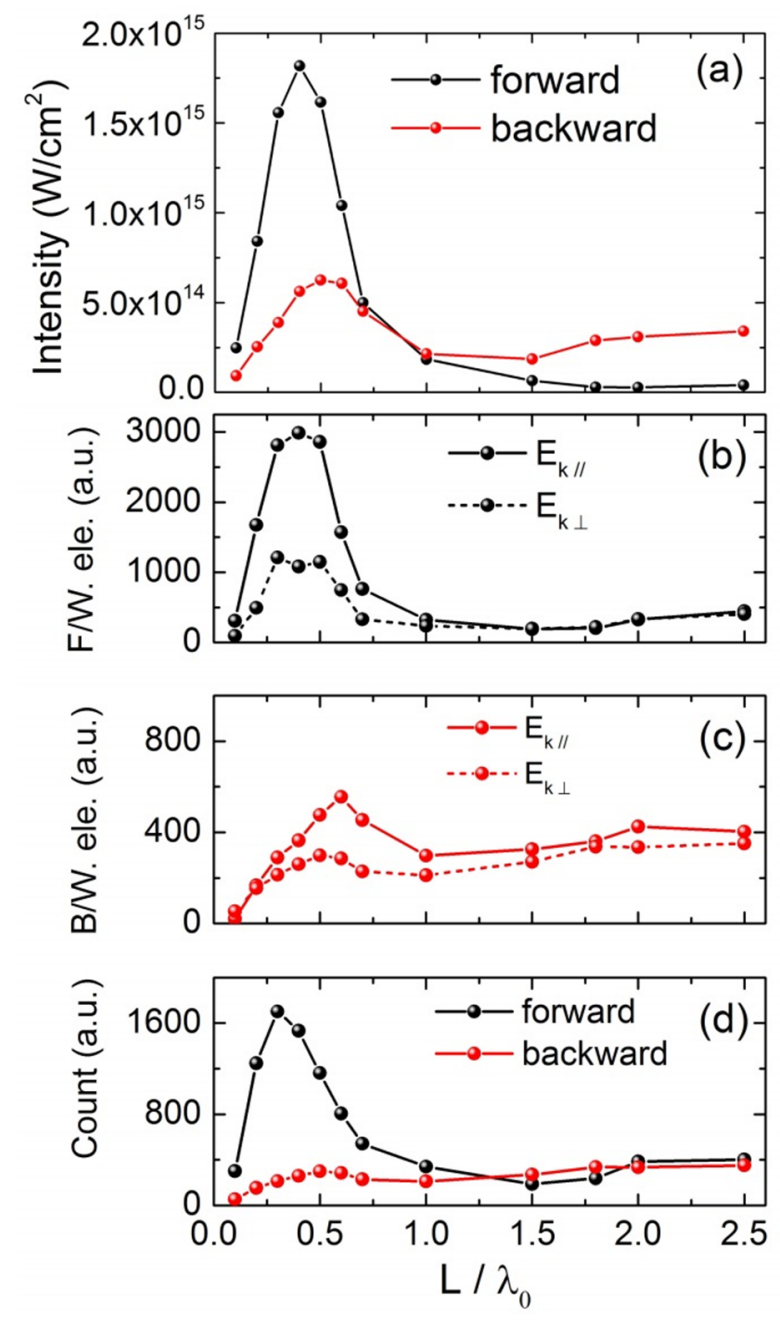

FIG. 7. (Color online) (a) Intensity of forward and backward $\mathrm{THz}$ radiations near the target surface as a function of the preplasma density scalelength; (b) and (c) show the total kinetic energy of forward and backward moving hot electrons, respectively, as a function of the density scalelength, where $E_{k \|}$ and $E_{k \perp}$ represent longitudinal and transverse kinetic energy, respectively; and (d) Total number of hot electrons as a function of density scalelength. Incident angle $\theta=45^{\circ}, a_{0}=0.6\left(I_{0}=7.7\right.$ $\times 10^{17} \mathrm{~W} / \mathrm{cm}^{2}$ ). Target thickness $D=8 \mu \mathrm{m}$.
As discussed earlier, the number of electrons is the key parameter in generating strong CTR. The charge of the hot electrons is calculated with the laser plasma interaction length in the $\mathrm{z}$ direction estimated as $6.6 \mu \mathrm{m}$ (FWHM of laser spot). The total charges of forward and backward electrons faster than $0.1 \mathrm{MeV}$ are $6.8 \mathrm{nC}$ and $1.6 \mathrm{nC}$, respectively. Because of the huge charges, the $\mathrm{THz}$ radiation can be as high as hundreds of $\mathrm{MV} / \mathrm{cm}$, even though most hot electrons have a moderate energy around $1 \mathrm{MeV}$ when the laser intensity is at the order of $10^{18} \mathrm{~W} / \mathrm{cm}^{2}$. From the radiation intensities in the optimal conditions, estimation on the energy conversion efficiency can be made, which are at least $0.2 \%$ and $0.08 \%$ for the forward and backward radiations, respectively.

It also can be seen from Fig. 7(a), as the scalelength increases beyond $\lambda_{0}$, the intensity of forward $\mathrm{THz}$ radiation reduces very quickly. On the other hand, the backward radiation decreases slightly and stays at a level about $60 \%$ of the maximum intensity over a wide range of scalelength (for range $L / \lambda_{0}>1$ ). When there is a large volume of underdense plasma involved ${ }^{29}$, the dominant mechanism of hot electrons generation changes from resonance absorption to two-plasmon decay and ponderomotive force acceleration with a reduced number of hot electrons.

Figures 7(b) and 7(c) illustrate the total kinetic energy $E_{\mathrm{k}}$ for all hot electrons with $E_{\mathrm{k}} \geq 0.2 \mathrm{MeV}$ produced under different preplasma density scalelengths. As expected, the optimal scalelength for $\mathrm{THz}$ radiation and for hot electron generation agree with each other. Both longitudinal and transverse kinetic energy of forward and backward electrons are plotted. According to Fig. 7(b), at $L=0.4 \lambda_{0}$ where resonance absorption dominates, the longitudinal kinetic energy of forward electrons is much higher than the transverse kinetic energy. Comparing Figs. 7(b) and 7(d), it is found that the number and total energy of forward electrons are much greater than backward electrons. When the scale of preplasma is in medium size, such as $L=2 \lambda_{0}$, the total energy of hot electrons is slightly increased, possibly due to ponderomotive force acceleration and the development of the two-plasmon decay instability. As a result, the 
backward $\mathrm{THz}$ radiation shown in Fig. 7(a) increases slightly for $L>\lambda_{0}$. However, the increase is not observed for the forward radiation, since hot electrons need to propagate through the target and therefore lose a considerable amount of energy when they reach the rear surface of the target. Because the number of forward hot electrons is much more than backward ones according to Fig. 7(d), the intensity of forward radiation can be 3 times of the backward radiation.

In order to show the behavior of hot electrons under different preplasma conditions, temporal evolution of the kinetic energy of the hot electrons is plotted in Fig. 8. When $L=0.4 \lambda_{0}$ as in Fig. 8(a), more electrons are accelerated in the forward longitudinal direction. Interestingly, there are two peaks for the longitudinal kinetic energy for both the forward and backward moving electrons (shown by the solid lines). The first peaks are attributed to the primary forward and backward hot electrons driven by the laser interaction. The second peaks are found at later times as results of transport of the first peaks through the following processes.

When the first bunch of forward electrons reaches the rear side of the target, they will be dragged back into the target by the strong sheath field formed at the rear side, and then form the second bunch of backward electrons. When these backward electrons are dragged back by the sheath field formed at the front of the target, they re-enter the target and form the second bunch of forward electrons. Both second forward and backward electrons stem from the primary forward electrons ${ }^{14}$. These are known as the hot electron refluxing processes ${ }^{30}$. At long preplasma scale length $L=2.0 \lambda_{0}$, Fig. 8(b) shows temporal profiles of hot electron energy. In this case, the kinetic energies for the forward and backward electrons are comparable, and are much smaller than those found at the small scalelength of $L=0.4 \lambda_{0}$. The effect of hot electron refluxing becomes much weaker in this case, so that the second peaks of hot electron flux are not as distinct as in the small scalelength case.
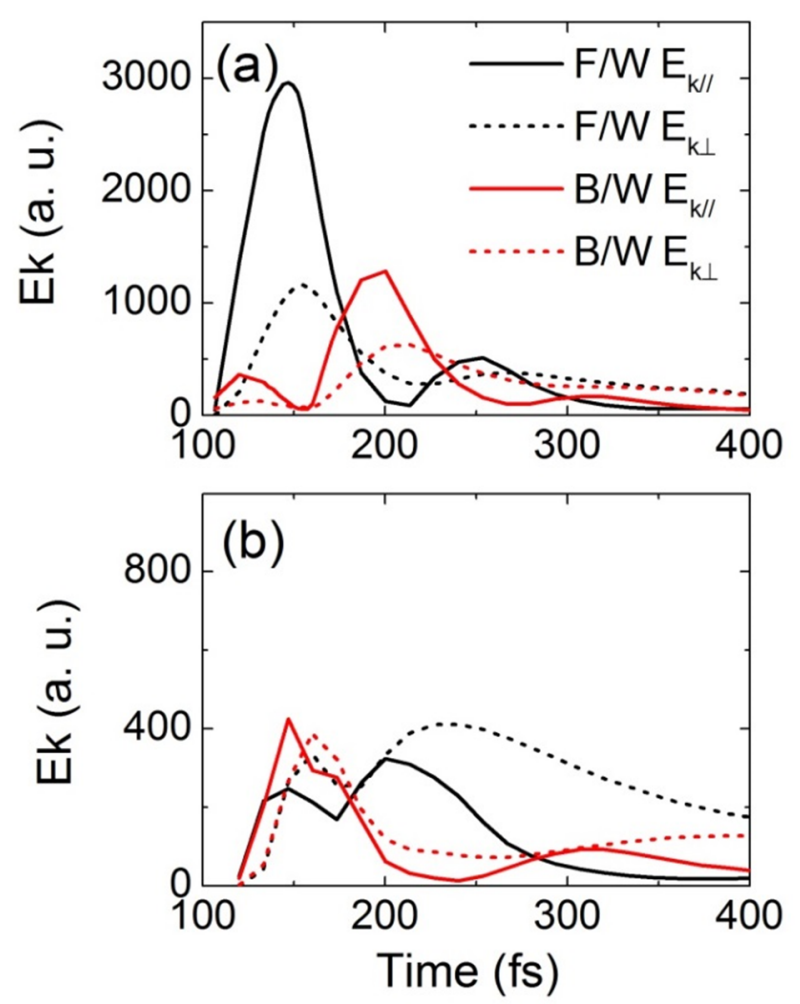

FIG. 8. (Color online) Temporal evolution of total longitudinal and transverse kinetic energy of forward and backward hot electrons (with $E_{\mathrm{k}}>0.2 \mathrm{MeV}$ in and near the target). (a) $L=0.4 \lambda_{0}$; (b) $L=2.0 \lambda_{0}$. Other parameters follow Fig. 3.

Simulations with normal incidence of laser are performed, where hot electron production due to resonance absorption and vacuum heating is excluded. In this case, hot electrons are mainly produced by ponderomotive force acceleration and the two-plasmon decay instability. Figure 9 shows that both forward and backward $\mathrm{THz}$ radiations increase with the scalelength of the preplasma. The kinetic energy and number of the hot electrons show similar dependence. When $L>2 \lambda_{0}$, the intensity of the forward radiation decreases slightly, due to the energy loss of hot electrons after the long distance of propagation. In the meantime, the intensity of the backward radiation increases monotonically in the range of interest.

It is worthwhile to point out that the CTR efficiency does not only depend on the hot electron number, but also on the density ramp. Sharp gradient of optical dielectric 
constants of the target at boundaries is more favorable for generation of transition radiation. A shorter length of preplasma implies a larger gradient of plasma density. As an example, we compare the backward $\mathrm{THz}$ emissions in Fig. 7(a) when $L=0.5 \lambda_{0}$ and $1.5 \lambda_{0}$. The emission is evidently stronger when the preplasma is shorter, even though the total energy and number of backward hot electrons are similar in these two cases.
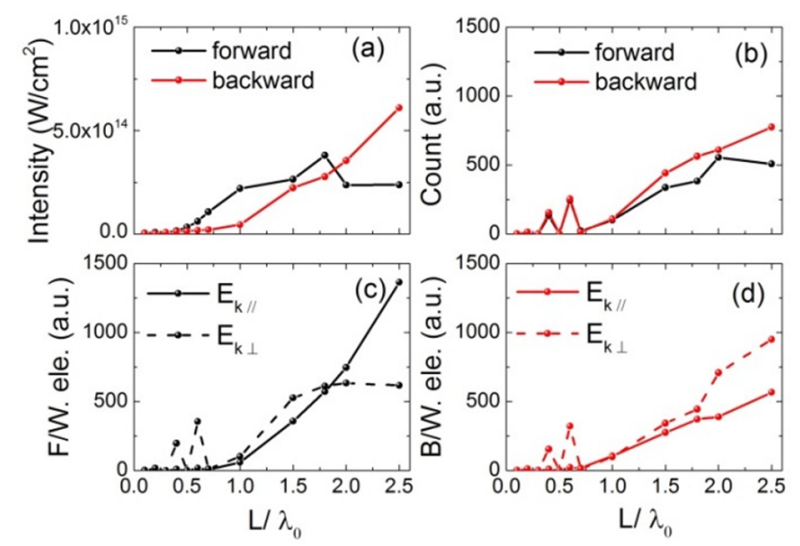

FIG. 9. (Color online) Correlation of $\mathrm{THz}$ radiation and hot electron production under normal incidence of laser pulses and different preplasma plasma conditions. (a) $\mathrm{THz}$ radiation intensity; (b) Number of hot electrons; (c) Kinetic energy of forward hot electrons; (d) Kinetic energy of backward hot electrons. Other parameters follow Fig. 3.

\section{Effects of target thickness and density}

The forward $\mathrm{THz}$ radiation from the target back will not be observed when the target is thick, because hot electrons deplete their energy during their transport in plasma. If a thin target is used, not only forward $\mathrm{THz}$ radiation is produced, second pulses of forward and backward $\mathrm{THz}$ radiations are also emitted. Since forward hot electrons carry much higher energy than the backward ones, the second pulse of the backward radiation (produced by the refluxing of forward electrons) can be stronger than the first backward pulse. Figure 10 shows the radiation intensity and electron kinetic energy of the first pulse as functions of the target thickness. Note that only the quantities associated with the first pulse is of interest, as they are produced directly by the laser interaction. As expected, the thickness of the target does not change the first pulse of the backward $\mathrm{THz}$ radiation (red line). However, for the generation of forward $\mathrm{THz}$ radiation (black line), an optimal thickness of the target is found at about $8 \lambda_{0}$ in Fig. 10(a), where a $20 \%$ increase of $\mathrm{THz}$ radiation is found as compared to the case of very thin targets. This can be explained as follows.

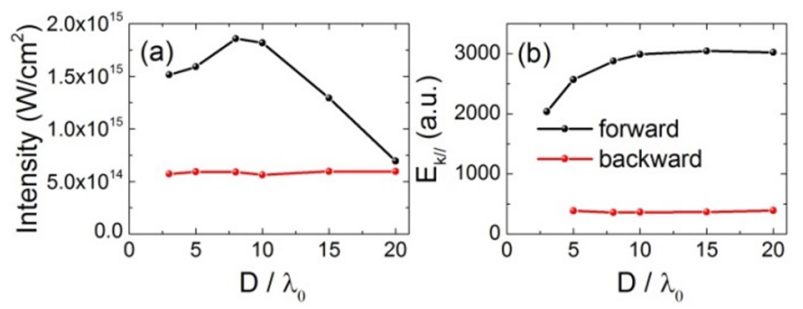

FIG. 10. (Color online) THz radiation intensity and hot electron kinetic energy as a function of the target thickness. (a) Forward and backward $\mathrm{THz}$ radiation intensity; (b) Kinetic energy of forward and backward hot electrons. Other parameters follow Fig. 3.

After the forward hot electrons produced at the target surface by laser preplasma interaction, they transport into the target and deposit part of their energy to the background electrons, making more hot electrons. For transition radiation, both the energy of a single electron and the number of electrons are important. In a thin target, the distance is too short for hot electrons to deposit their energy to make more hot electrons, where as in a thick target, the hot electrons will lose too much of their energy during the long propagation. As a result, there is an optimal distance that the energy and number of hot electrons are most advantageous to the generation of transition radiation. Since the electron energy depends on the laser intensity, the optimal thickness depends on laser intensity, too. Note that the primary backward hot electrons at $D=3 \lambda_{0}$ in Fig. 10(b) is not plotted, because the target is too thin that the primary backward electrons overlap with the returning ones and is therefore difficult to distinguish them.

Besides the parameters we have discussed earlier, the effect of maximum plasma density on the generation 
of $\mathrm{THz}$ radiations is also considered. As the maximum plasma density is changed from $10 n_{\mathrm{c}}$ to $110 n_{\mathrm{c}}$ while keeping other parameters fixed, there is no obvious change observed in the intensity or profile of the $\mathrm{THz}$ radiations. On the other hand, when different target materials are used in experiments, all laser plasma interaction parameters will be changed, including the maximum plasma electron density and preplasma conditions. Therefore, the generated $\mathrm{THz}$ radiation may be considerably different.

\section{E. Comparison with some experimental observations}

We have found the profiles and properties of $\mathrm{THz}$ radiation in some experiment reports match well with the CTR mechanism. For example, Gopal et al. ${ }^{7}$ reported recently a forward $\mathrm{THz}$ radiation of over $460 \mu \mathrm{J}$ in the 0.1 $30 \mathrm{THz}$, obtained from a $5 \mu \mathrm{m}$ thin metal foil target irradiated with laser pulses with focused intensity up to 5 $\times 10^{19} \mathrm{~W} / \mathrm{cm}^{2}$. It is found that the $\mathrm{THz}$ radiation yield is proportional to the square of particle number. The $\mathrm{THz}$ emission from the target back decreases with the target thickness. It is also shown by the same group ${ }^{31}$ that the measured $\mathrm{THz}$ radiation is distributed broadly in space, which is weakest at the center and increases with distance away from the center. All these properties agree with CTR depicted above. By using lasers with different polarizations and contrast ratios, Li et al. ${ }^{6,32,33}$ revealed the significance of hot electrons, especially the role of resonance absorption, to the generation of $\mathrm{THz}$ radiation.

The dependence of $\mathrm{THz}$ radiation intensity on the contrast ratio observed in their experiments agrees with Fig. 7(a), where an optimal density scalelength exists. Note that the transverse kinetic energy (proportional to surface currents) depends relatively weakly on the plasma density scalelength. In an earlier work, Sagisaka et al. ${ }^{34}$ reported experimental studies on $\mathrm{THz}$ radiation where a $2 \times 10^{17} \mathrm{~W} / \mathrm{cm}^{2}$ laser is used. It is found that the $\mathrm{THz}$ radiation does not show a strong directionality. This is reasonable if it is produced by the CTR mechanism, because the average kinetic energy of hot electrons under the given laser intensity is generally at tens of $\mathrm{keV}$. According to the transition radiation distribution shown in Fig. 1(a), the radiation will spread broadly in space.

\section{SUMMARY}

We have reported a mechanism of strong $\mathrm{THz}$ sources produced by ultrashort high intensity laser interaction with solid targets via coherent transition radiation. The generation of hot electrons is found to be a critical factor for this mechanism. When the density scalelength of the preplasma is optimized (e.g., by proper control of laser contrast ratio), the peak $\mathrm{THz}$ radiation field can reach $400 \mathrm{MV} / \mathrm{cm}$, although the driving laser is at a moderate intensity of $7.7 \times 10^{17} \mathrm{~W} / \mathrm{cm}^{2}$. The energy conversion efficiencies can be as high as over $0.2 \%$ and $0.08 \%$ for the first pulses of forward and backward radiations, respectively. The coherent transition radiation mechanism can explain some of previous experimental observations of $\mathrm{THz}$ radiation. It is expected that the $\mathrm{THz}$ radiation discussed in this paper can be used not only as a high power $\mathrm{THz}$ sources, but also as a useful diagnostic of relativistic laser-solid interaction and hot electron transport.

\section{ACKNOWLEDGMENTS}

This work was supported by the A*STAR Computational Resource Centre through the use of its high performance computing facilities. The work by Z.M.S. is supported in part by the National Basic Research Program of China (No. 2014CB339801), the National Science Foundation of China (Nos. 11421064 and 11374210), a MOST international collaboration project (No. 2014DFG02330), and a Leverhulme Trust Research Grant.

\section{References}

1. G. P. Williams, Rep. Prog. Phys. 69, 301 (2006); K. Reimann, Rep. Prog. Phys. 70, 1597 (2007).

2. H. G. Roskos, M. D. Thomson, M. Kreß, and T. Löffler, Laser \& Photon. Rev. 1, 349 (2007).

3. D. H. Auston, K. P. Cheung, J. A. Valdmanis, and D. A. Kleinman, Phys. Rev. Lett. 53, 1555 (1984). 
4. D. Daranciang, J. Goodfellow, M. Fuchs, H. Wen, S. Ghimire, D. A. Reis, H. Loos, A. S. Fisher and A. M. Lindenberg, Appl. Phys. Lett. 99, 141117 (2011).

5. Y. Gao, T. Drake, Z. Chen, and M. F. DeCamp, Opt. Lett. 33, 2776 (2008).

6. Y. T. Li, C. Li, M. L. Zhou et al., Appl. Phys. Lett. 100, 254101 (2012).

7. A. Gopal, S. Herzer, A. Schmidt et al., Phys. Rev. Lett. 111, 074802 (2013).

8. K. Y. Kim, J. H. Glownia, A. J. Taylor, and G. Rodriguez, Opt. Express 15, 4577 (2007).

9. H. C. Wu, J. Meyer-ter-Vehn, and Z. M. Sheng, New J. Phys. 10, 043001(2008).

10. M. Chen, A. Pukhov, X.-Y. Peng, and O. Willi, Phys. Rev. E 78, 046406 (2008).

11. W.-M. Wang, P. Gibbon, Z.-M. Sheng, and Y.-T. Li, Phys. Rev. Lett. 114, 253901 (2015).

12. Z. M. Sheng, K. Mima, J. Zhang, and H. Sanuki, Phys. Rev. Lett. 94, 095003 (2005).

13. H. C. Wu, Z. M. Sheng, Q. L. Dong, H. Xu, and J. Zhang, Phys. Rev. E 75,016407 (2007).

14. W. J. Ding, Z. M. Sheng, and W. S. Koh, Appl. Phys. Lett. 103, 204107 (2013).

15. J. Zheng, K. A. Tanaka, T. Miyakoshi, Y. Kitagawa, R. Kodama, T. Kurahashi, and T. Yamanaka, Phys. Plasmas 10, 2994 (2003).

16. Z. Wu, A. S. Fisher, J. Goodfellow, M. Fuchs, D. Daranciang, M. Hogan, H. Loos, and A. Lindenberg, Rev. Sci. Instrum. 84, 022701 (2013).

17. I. M. Frank and V. L. Ginzburg, J. Phys. USSR 9, 35 (1945); V. L. Ginzburg and I. M. Frank, Zh. Eksp. Teor. Fiz. 16, 15 (1946).

18. Z. M. Sheng, Y. Sentoku, K. Mima, J. Zhang, W. Yu, and J. Meyer-ter-Vehn, Phys. Rev. Lett. 85, 5340 (2000); M. Chen, Z. M. Sheng, and J. Zhang, Phys. Plasmas 13, 014504 (2006).

19. W. J. Ding, Z. M. Sheng, J. Zhang, and M. Y. Yu, Phys. Plasma 16, 042315 (2009).
20. Y. Q. Cui, W. M. Wang, Z. M. Sheng, Y. T. Li, and J. Zhang, Plasma Phys. Controlled Fusion 55, 085008 (2013).

21. Y. T. Li, X. H. Yuan, M. H. Xu, Z. Y. Zheng, Z. M. Sheng, M. Chen, Y. Y. Ma, W. X. Liang, Q. Z. Yu, Y. Zhang, F. Liu, Z. H. Wang, Z. Y. Wei, W. Zhao, Z. Jin, and J. Zhang, Phys. Rev. Lett. 96, 165003 (2006).

22. K. G. Estabrook, E. J. Valeo, and W. L. Kruer, Phys. Fluids 18, 1151(1975).

23. F. Brunel, Phys. Rev. Lett. 59, 52 (1987).

24. H. Ruhl, Y. Sentoku, K. Mima, K. A. Tanaka, and R. Kodama, Phys. Rev. Lett. 82, 743 (1999).

25. W. Yu, V. Bychenkov, Y. Sentoku, M. Y. Yu, Z. M. Sheng, and K. Mima, Phys. Rev. Lett. 85, 570 (2000).

26. Y. Ping, R. Shepherd, B. F. Lasinski et al., Phys. Rev. Lett. 100, 085004 (2008).

27. J. Zhang, Y. T. Li, Z. M. Sheng, Z. Y. Wei, Q. L. Dong, and X. Lu, Appl. Phys. B Lasers Opt. 80, 957 (2005).

28. D. W. Forslund, J. M. Kindek, K. Lee, E. L. Lindman, and R. L. Morse, Phys. Rev. A 11, 679 (1975).

29. H. Hamster, A. Sullivan, S. Gordon, W. White, and R. W. Falcone, Phys. Rev. Lett. 71, 2725 (1993).

30. P. Neumayer, B. Aurand, M. Basko, B. Ecker, P. Gibbon et al., Phys. Plasmas 17, 103103 (2010).

31. A. Gopal, T. May, S. Herzer et al., New J. Phys. 14, 083012 (2012).

32. C. Li, M. L. Zhou, W. J. Ding et al., Phys. Rev. E 84, 036405 (2011).

33. C. Li, Y. Q. Cui, M. L. Zhou et al., Opt. Express 22, 11797 (2014).

34. A. Sagiska, H. Daido, S. Nashima et al., Appl. Phys. B 90, 373 (2008). 